\title{
Compétences et expertise professionnelle de l'architecte dans le travail de conception
}

\author{
Dominique Raynaud ${ }^{1}$
}

Résumé : Certains travaux récents envisagent de transposer à l'architecture les résultats de l'anthropologie des sciences et techniques. L'article examine chaque point de la transposition. À la faveur des nouvelles recherches, on doit admettre une part de négociation dans la conception, et le caractère révisable des compétences professionnelles. Mais, en maximalisant ces conclusions, certains travaux parviennent à des résultats discutables. La dérive ne fait pas du projet un phénomène totalement imprévisible ; l'accent mis sur la négociation peut occulter d'autres rapports sociaux, comme la contrainte ou la communication d'informations ; les compétences des acteurs ne sont pas interchangeables à merci. L'échec de la transposition est explicité par une comparaison des situations de conception en architecture et dans l'industrie. Le fait que le bâtiment soit une production unique et située le soumet à des contraintes de site. Connaissables ex ante, ces contraintes expliquent pourquoi la conception demeure le noyau dur de l'expertise professionnelle de l'architecte, en dépit des succès rencontré par le thème de la négociation.

Mots-clefs : conception architecturale, négociation, compétences professionnelles.

Abstract : Recent works try to transpose the results of the anthropology of science and technics to architecture. This paper reviews every point of this transposition. Thanks to new researches, we have to admit the reality of projects' drift, the existence of a part of negociation in design, and the revisable nature of professional skills. But some works, maximizing these findings, reach questionable results. Drift does not turn project into a wholly unexpected process; negociation can hide other important social relations (as constraint or communication); actors' skills are not arbitrarily interchangeable. The failure of the transposition is then clarified by a comparison of architecture and industry design situations. Building being an only and setlled production, it is subjected to specific site constraints. Knowable ex ante, those constraints explain why design remains the hard core of architect's professional expertise, in spite of the increasing success of negociate activities.

Keywords : architectural design process, negociation, professional skills.

\footnotetext{
${ }^{1}$ Département de Sociologie, Université Pierre-Mendès-France, BP 47, 38040 Grenoble cedex 9, adresse électronique : dominique.raynaud@upmf-grenoble.fr.
} 


\section{Introduction}

L'étude de la conception architecturale, inaugurée par les recherches anglo-saxonnes (Simon, 1974² ; Alexander, 1971), est restée pendant deux décennies un domaine d'étude spécialisé des architectes. Les années 1980 et 1990 ont été marquées par une forte croissance des publications sur ce thème. On a assisté parallèlement à une ouverture des points de vue disciplinaires traitant de la conception, la sociologie (du travail, des professions, des sciences et techniques) étant largement représentée depuis les années 1990. Cette multiplication des points de vue a suscité des débats productifs entre internalistes - (Boudon, 1992, 1994, 1995 ; Conan, 1981, 1990 ; Prost, 1992, 1995 ; Raynaud, 1999) — et externalistes — (Toussaint et Younès, 1997 ; Champy, 1998, 1999 ; Tapie, 1999, 2000). Ces termes d'internalisme et d'externalisme, qui sont issus de l'épistémologie et de l'histoire des sciences ${ }^{3}$, qualifient, relativement à la conception, les travaux qui explorent les actes de conception sur un plan cognitif (internalisme), et ceux qui envisagent la conception comme un lieu d'interactions sociales entre acteurs du projet (externalisme). L'intérêt du débat tient à ce que ces positions apportent souvent des éclairages complémentaires sur les tâches de conception, l'une mettant en évidence la façon dont les données et contraintes initiales du projet se construisent, l'autre, par quelles opérations mentales les concepteurs se servent de ces données et contraintes pour parvenir à une proposition architecturale acceptable.

Certains textes de sociologie, écrits à la faveur de synthèses rapides ${ }^{4}$, ont occasionné des

\footnotetext{
${ }^{2}$ On lui doit d'avoir mis en évidence certains processus de pensée spécifiques aux concepteurs. L'essai sur « La science de la conception » (Simon, 1974, pp. 73-103) montre que la conception architecturale est un domaine à solutions sous-optimales ou satisficing (le concepteur n'ayant en général, ni le temps, ni les ressources cognitives pour accéder à la solution optimale, à supposer que celle-ci existe) ; et un domaine d'heuristiques (le concepteur, qui ne peut appliquer un algorithme ou faire l'inventaire des solutions possibles, doit en général imaginer des méthodes de résolution). Cette perspective a été largement développée en France (Boudon, 1992, 1994 ; Conan, 1981, 1990 ; Prost, 1992, 1995).

${ }^{3}$ Georges Canguilhem les définit ainsi : «L'externalisme [...] conditionne un certain nombre d'événements par leurs rapports avec des intérêts économiques et sociaux, des exigences et des pratiques techniques, avec des idéologies religieuses ou politiques. L'internalisme [...] se place à l'intérieur-même de l'oeuvre scientifique pour en analyser les démarches par lesquelles elle cherche à satisfaire aux normes spécifiques qui permettent de la définir comme science » (Canguilhem, 1968, p. 15).

${ }^{4}$ Pour écrire le premier article, l'auteur dit avoir « consulté l'ensemble des travaux financés par le Bureau de la recherche architecturale sur le thème de la conception en architecture » (Callon, 1996, p. 26). Quant au deuxième article, tiré des actes du séminaire Euro-conception, il propose une simple transposition à l'architecture
} 
réactions de la part des praticiens ou des chercheurs sur la conception architecturale. Les positions sont en désaccord sur :

[1] la possibilité d'une transposition des modèles d'organisation du domaine de l'industrie à l'architecture ${ }^{5}$, dissensus dont découlent trois sous-problèmes touchant respectivement :

[1a] à la nature imprévisible du projet architectural,

[1b] au caractère central des processus de négociation dans les activités de conception,

[1c] à l'étendue et à l'existence des compétences cognitives spécifiques du concepteur.

La question de la transposition à l'architecture des modèles élaborés ailleurs ne pouvant être jugée a priori, la réponse devra découler d'un examen détaillé des problèmes [1a], [1b], [1c], et d'une analyse comparative des situations de conception dans l'industrie et en architecture $^{6}$.

Cet article n'a pas d'autre objectif que de parvenir à une réduction de la discordance observée sur chacun des points cités (caractère imprévisible du projet, importance de la négociation, mise en doute des compétences de l'architecte).

\section{Le caractère imprévisible du projet}

Le phénomène de la dérive des projets d'architecture, présenté dans diverses publications récentes (Conan, 1990, 1995), exprime le fait que les choix initiaux de l'architecte peuvent être modifiés, dans la phase de conception, par l'apparition de données, contraintes ou possibilités d'action, inconnues ou négligées à une date antérieure par les concepteurs. Ainsi, pour le concours de l'Institut du Monde arabe, lancé en 1981, Jean Nouvel et ses associés n'ont pas tenu compte du gabarit urbain imposé par le règlement d'urbanisme. Ayant proposé une

\footnotetext{
des modèles tirés du domaine industriel.

${ }^{5}$ L'auteur conclut : « Les résultats de l'anthropologie des sciences et des techniques sont ici parfaitement transposables » (Callon, 1996, p. 33).

${ }^{6} \mathrm{Je}$ m'appuie, pour conduire cet examen, sur ma propre pratique d'architecte, acquise en agence entre 1984 et 1992, sur ma participation aux travaux du Laboratoire d'Architecturologie et de Recherches sur l'Épistémologie de l'Architecture (composante du GNRS UMR 7544), et sur quelques questions complémentaires posées dans cinq agences d'architecture, notamment sur l'organisation des tâches de conception.
} 
esquisse où le bâtiment avait une côte d'altitude de $38 \mathrm{~m}$, ils ont ensuite dû réduire de $6 \mathrm{~m}$ la hauteur du bâtiment (en diminuant la hauteur de l'étage courant), de manière à respecter le gabarit prescrit.

C'est probablement en ayant en vue des exemples similaires que l'on utilise l'idée de dérive pour donner une vue générale du processus de conception : «Les choix sont en permanence remis sur le métier », ou bien : «Le bâtiment [...] est le résultat improbable et non voulu d'une lente dérive à laquelle ont contribué de manière imprévisible un grand nombre d'acteurs [...] Ce n'est qu'a posteriori que l'on peut parler d'un projet dont le bâtiment serait la réalisation » (Callon, 1996, pp. 26, 29). Chacune de ces assertions mérite un examen.

\subsection{L'absence de stabilisation des choix}

Le premier énoncé, qui expose le caractère instable et fluctuant des décisions du concepteur, est renforcé par l'affirmation que «Les révisions proposées peuvent être déchirantes et toucher indifféremment le choix des matériaux ou l'organisation générale du bâtiment [...] Le processus de création ou d'invention ne débute pas avec des ruptures inaugurales suivies de modifications de détails » (Callon, 1996, p. 26). Si l'on peut admettre qu'il existe, dans tout projet d'architecture, une part de dérive, la maximalisation de cette idée produit un résultat contre-intuitif qui pourrait être soumis à un test empirique.

Le degré de dérive des projets n'est pas nécessairement un objet d'appréciation qualitative. Il peut être éprouvé par diverses méthodes. Contentons-nous d'évoquer deux pistes. Les archives des agences d'architecture offrent la possibilité de comparer les représentations ex ante du bâtiment avec le bâtiment construit. Or, on constate régulièrement une bonne correspondance de la représentation et de l'ouvrage réalisé. Cela vaut évidemment lorsque le document graphique est un plan d'exécution - comparer le plan d'exécution et la photographie de la clinique dentaire Ark de Shin Takamatsu (Guillot, 1989, p. 95) - mais aussi pour les perspectives d'aspect - comparer la perspective et la photographie du Larkin Company Administration Building de Frank Lloyd Wright (Pfeiffer, 1990, p. 238 ; Riley, 1994, p. 143) - et c'est encore le cas de certains croquis d'étude - comparer le croquis et la photographie de l'Hôtel Salé (Simounet, 1996, pp. 113, 120). Ces vérifications montrent que, en général, le phénomène de dérive affecte, non pas l'organisation générale du bâtiment, mais des aspects secondaires du projet. 
Certaines classes de bâtiment suggèrent un recours à un protocole d'observation plus strict. Ainsi, les constructions immobilières exigeant un récolement des plans, il devient possible de mesurer systématiquement l'écart entre les PEO (plans d'exécution des ouvrages) et ceux du DOE (dossier des ouvrages exécutés). Une vérification sur quelques projets montre que les modifications introduites au moment du récolement constituent des ajustements de détail. Il existe donc une dérive des projets, que l'on ne saurait nier, mais celle-ci n'est pas assimilable à une refonte générale du projet d'architecture.

\subsection{Des résultats improbables et non intentionnels}

Le deuxième énoncé, faisant du bâtiment le « résultat improbable et non voulu d'une lente dérive à laquelle ont contribué de manière imprévisible un grand nombre d'acteurs » (Callon, 1996, p. 26), décrit l'impossibilité que les concepteurs auraient de maintenir l'idée directrice du projet. Le processus de conception serait caractérisé par des résultats involontaires et imprévisibles.

La simple correspondance entre les représentations ex ante et le bâtiment construit, dont il vient d'être question, permet de douter de cet énoncé. Jean-Charles Lebahar a montré par ailleurs que l'évolution des modifications de fond vers des ajustements de détail était la marque d'une « réduction d'incertitude » finalisée, pour l'obtention de laquelle le concepteur met en oeuvre une redondance de l'information entre le plan et les mentions écrites, entre les différentes vues du bâtiment, entre les plans et le descriptif, etc. (Lebahar, 1983). Quand au caractère involontaire ou non intentionnel du résultat du travail de conception, d'autres recherches (Boudon, 1992, 1994), montrent que les opérations de conception se déroulent dans un espace finalisé (la fin supposant un ajustement des moyens qui peut être parfois complexe, mais jamais improbable ou inattendu). Le caractère intentionnel des opérations de conception s'oppose à la thèse selon laquelle l'édifice serait le résultat non voulu d'une dérive incontrôlable.

\subsection{Le projet, conséquence du bâtiment}

Une conséquence de cette conception maximaliste de la dérive est qu'il ne serait possible de parler de projet qu'une fois celui-ci réalisé. Ainsi, les analyses classiques ne feraient que « renverser le cours des choses, transformer en cause ce qui est une conséquence » (Callon, 
1996, p. 29). Si l'on prend cette proposition à la lettre, il faut mettre le bâtiment en position de cause, et le projet en position de conséquence. Cette conclusion, qui se heurte à la description de la conception comme un " espace d'actions finalisées », soulève d'autres difficultés : si le projet est une conséquence du bâtiment, c'est que le bâtiment peut être construit sans projet. Or, dans le contexte actuel, et pour un bâtiment de complexité moyenne, combien d'entreprises accepteraient de construire un bâtiment sans plans d'exécution, sans descriptif et sans estimatif des travaux?

Conclusion : autant l'idée de dérive décrit un phénomène observable sur de nombreux projets, autant la maximalisation de cette idée conduit à des résultats empiriquement infondés.

\section{Le rôle de la négociation dans la conception}

Les récents travaux de sociologie de la conception insistent sur le caractère «négocié » de cette activité (Callon, 1996, 1997 ; Tapie, 2000). La fréquente occurence des mots « négociation » et « compromis » dans ces travaux, traduit certainement le fort engouement que suscite ce thème en sociologie générale (Callon et Latour, $1991^{7}$; Strauss, 1992 ; Reynaud, 1999 ; Thuderoz, 2000). La négociation peut être définie comme une activité sociale qui, partant d'un conflit d'intérêts entre deux ou plusieurs acteurs, tente de parvenir à un accord acceptable par tous. La forme courante de l'accord est le compromis. Les sociologues ont par ailleurs observé que la négociation induit une régulation de la vie sociale, qui dépasse les objectifs immédiats que s'assignent les acteurs dans une négociation (Thuderoz, 2000). Certains échanges entre l'architecte, ses collaborateurs ou partenaires satisfont à cette définition. Par exemple, lorsqu'un architecte fait une proposition au maître d'ouvrage susceptible de remettre en question le budget global, leurs échanges rentrent dans le champ de la négociation. L'architecte consentira sans doute à serrer le coût d'objectif, le maître d'ouvrage acceptera de revoir le niveau des prestations, de sorte qu'ils parviendront à une solution de compromis, intermédiaire entre leurs objectifs initiaux.

Les architectes ont eu accès au caractère négocié des activités de conception par certaines

\footnotetext{
${ }^{7}$ Notons que cet engouement semble provenir d'une convergence de vues entre la sociologie du travail et la sociologie des sciences et techniques. Il est probable que cette idée de négociation soit encore une transposition de résultats obtenus en sociologie des sciences : « Négocier des connaissances signifie presque toujours en modifier le contenu [...] Se fabriquent ainsi des compromis qui affectent le contenu même des connaissances » (Callon et Latour, 1991, p. 30).
} 
recherches américaines, qui ont étudié les processus de négociation collective en architecture à partir d'une enquête sur plusieurs agences américaines (Cuff, 1982). Les recherches internalistes françaises mentionnent parfois des éléments de négociation dans la phase de conception (Conan, 1990, pp. 123-134 ; Boudon et al., 1994, p. 55). La difficulté ne se trouve donc pas dans le fait qu'il existe une part de négociation dans le travail de conception : c'est un résultat connu de tous. La difficulté se trouve plutôt dans le fait que l'engouement actuel à percevoir le caractère négocié des interactions entre l'architecte et ses partenaires finit par réduire le spectre des échanges observables : car il existe d'autres types de rapports sociaux que la négociation (libéralité, conflit, contrainte, obéissance, etc.) ; et il se peut fort bien que de tels rapports apparaissent au cours de la phase de conception. Il ne faut donc pas exclure que, lorsque certains auteurs avancent que «négociation » et « compromis » constituent les traits saillants de la conception architecturale, on ait affaire à une description partielle des activités de conception. On s'en rendra compte à la suite de deux développements.

\subsection{Modèle hiérarchique et modèle négocié}

On percevra tout d'abord une difficulté en comparant ${ }^{8}$ le contenu des certains articles consacrés au travail de conception en architecture (Callon, 1996, 1997). Dans le premier article, l'auteur traite de la négociation comme d'un fait incontournable structurant l'organisation de la conception architecturale, alors que, dans le second, il ne présente une comparaison des modèles négocié et hiérarchique que pour stimuler la réflexion des architectes et des urbanistes. Il se limite alors à exposer les résultats d'études effectuées dans l'industrie. Contrairement à la vision classique que propose le « modèle hiérarchique », le « modèle négocié » suppose que les compétences sont en permanence redistribuées entre les acteurs ${ }^{9}$. La question demeure de savoir pourquoi deux articles quasi-contemporains

\footnotetext{
${ }^{8}$ Cette comparaison est légitime. En effet, les mêmes conclusions sont tirées de l'existence des relations de négociation. On rapprochera par exemple : « Dans le modèle de la conception négociée, le projet n'est connu qu'à la fin » de : «Ce n'est qu'a posteriori que l'on peut parler d'un projet, dont le bâtiment serait la réalisation » (Callon, 1996 p. 29, 1997, p. 90).

${ }^{9}$ On trouve l'origine de ces notions de « modèle négocié » et de « modèle hiérarchique » dans les travaux sur le management de l'innovation (Burns et Stalker, 1966). En outre, dans une intéressante étude de 13 laboratoires de recherche industrielle (512 personnes interrogées), Terry Shinn démontra que les laboratoires de chimie et d'informatique avaient des structures organisationnelles très différentes (Shinn, 1980). Reprenant la terminologie des deux sociologues britanniques, l'auteur décrit celles-ci sous le nom de « mechanistic structures »
} 
apportent une réponse différente au problème de l'organisation des tâches de conception. L'analyse empirique de la conception en architecture concorde mieux avec la deuxième version. Chacun connaît en effet des agences d'architecture fonctionnant selon l'un ou l'autre modèle. Celle de Foster Associates se rapproche du modèle hiérarchique : la compartimentalisation des tâches, la séquentialité du processus de conception, les relations de type formel sont prépondérantes (Tric, 1999, p. 219). Au contraire, celle du gênois Renzo Piano hérite davantage du modèle négocié : chaque concepteur est multi-tâches, la séquentialité y est réduite au minimum, et les relations sont plutôt de type informel. Mais si, tant dans l'industrie que dans les agences d'architecture, on reconnaît l'existence de ces deux modèles d'organisation, pourquoi décrire l'organisation de la conception en architecture sous l'exclusive du « modèle négocié »? Ce rapprochement suggère que la négociation n'est peut être pas un trait central de la conception architecturale.

\subsection{D'autres types d'interaction sociale}

L'analyse empirique des interactions sociales entre les acteurs du projet architectural révèle la justesse des analyses en termes de négociation sur certains types d'échange, mais aussi les insuffisances de ce modèle à décrire l'ensemble des interactions de conception. Il existe en effet une diversité de rapports dont l'idée de négociation ne peut à elle seule rendre compte. Considérons seulement deux exemples.

Il existe, dans les activités de conception en architecture, des rapports de contrainte qui ne se ramenent pas au cadre de la négociation. Lorsque l'architecte conçoit un ERP (édifice recevant du public), il est tenu d'appliquer le Code de la Construction, lequel prescrit que, «Dans un établissement recevant du public, un éclairage de sécurité doit être prévu dans tous les cas » (Article R 123-8). L'injonction faite au concepteur constitue alors une contrainte, et si l'architecte omet de présenter le plan d'éclairage de sécurité, l'avis de la Commission de sécurité entraînera le refus du permis de construire. Quand bien même l'architecte prendrait contact avec elle pour lui soumettre une proposition, les échanges entre le concepteur et le représentant de l'État seraient encore placés sous le signe de la contrainte, non pas sous celui

versus « organic structures ", qui correspondent à peu près au « modèle hiérarchique » et au " modèle négocié » dont il est ici question. Selon Terry Shinn, ces différences organisationnelles seraient en partie héritées de la formation spécifique des ingénieurs (Grandes Écoles versus Université). 
de la recherche d'un compromis. En effet, l'éclairage de sécurité ou l'accessibilité des ERP aux handicapés ne sont pas des objets négociables. Ainsi, l'étude des contraintes de la conception architecturale (Raynaud, à paraître) échappe entièrement au cadre de la négociation.

Ces échanges entre le concepteur et ses interlocuteurs ne sont d'ailleurs pas prisonniers d'une alternative négociation versus contrainte. Le géomètre fait état au concepteur de certaines contraintes topographiques qu'il devra prendre en compte (forme et dimensions du terrain, orientation, relief, position d'arbres ou d'édifices à conserver, etc.) Mais les rapports entre architecte et géomètre ne relèvent d'aucune forme de négociation (le géomètre n'a pas le pouvoir de faire évoluer les caractères topographiques de la parcelle, pas plus qu'il n'a le pouvoir de faire évoluer les décisions de l'architecte). Leurs échanges ne sont pas davantage descriptibles par une relation de contrainte, car la contrainte se définit par une « menace de sanction » (Nozick, 1991 ; Neuberg, 1990). Ne pouvant exercer de sanction, le géomètre ne saurait contraindre l'architecte à adopter telle ou telle solution architecturale. Leurs échanges sont simplement caractérisés par la demande et la communication d'informations.

Les architectes connaissent, depuis quelques années, des typologies explorant la diversité des échanges entre les acteurs de la conception. En étudiant l'influence des NTIG (nouvelles technologies de l'information et de la communication) et des EDI (échanges de données informatisées) sur les pratiques de conception, certaines recherches ont envisagé une telle typologie des échanges (Halin et al., 1998 ; Bignon et al., 1999 ; Malcurat et al., 2000ab). Notons au préalable que ces auteurs, en posant que « les réseaux [informatiques] induisent le passage d'une ingénierie fortement discrétisée à une ingénierie de plus en plus simultanée » (Bignon et al, 1999), adoptent un cadre de réflexion proche du modèle négocié. Mais leur analyse va bien au-delà de la découverte de ces relations de négociation. Ils ne se contentent pas de percevoir des flux d'échange indifférenciés entre les acteurs : ils fournissent un modèle pour étudier le type et le contenu des échanges entre les partenaires du projet architectural. Les auteurs définissent ainsi un certain nombre de « requêtes typées » ou de «briques de coopération » (Bignon et al., 1999), dont les principales sont :

La brique " pour information " : l'émetteur met à disposition une information présentant un intérêt pour le processus de conception mais dont l'absence ne lui nuit pas fondamentalement.

La brique "pour consultation" : l'émetteur met à disposition une information nécessaire au projet et qui exige d'être lue. 
La brique "pour question » : l'émetteur demande un conseil, des précisions ; il attend une réponse en retour.

La brique "pour addition " : l'émetteur met à disposition un document partiel devant être complété et retourné.

La brique "pour correction " : l'émetteur met à disposition un document et en attend des commentaires et des annotations.

La brique "pour validation " : l'émetteur demande l'approbation de l'information qu'il transmet. Son retour est obligatoire pour la poursuite du processus de conceptionconstruction (Bignon et al., 1999).

Ces analyses (Halin et al., 1998 ; Bignon et al., 1999 ; Malcurat et al., 2000 a et b) ou celles, convergentes, d'autres auteurs (Tata, Casals, Godart, 1999), montrent que les échanges entre l'architecte et ses partenaires ne répondent ni à des objectifs uniformes, ni à des modalités de réalisation identiques. Certains ont reconstruit, sur un projet type, les interactions entre architecte, urbaniste, ingénieur structure et ingénieur fluides (Halin et al., 1998). Selon les auteurs, les rapports entre l'architecte et l'ingénieur fluides relèvent d'un paradigme contractant / prestataire : le second ne s'engage dans le projet que sur la base de plans élaborés en amont par l'architecte. Les échanges entre l'architecte et l'ingénieur structure relèvent quant à eux d'un paradigme coauteur / coauteur : l'ingénieur ajoute de nouvelles informations au plan de l'architecte, lesquelles peuvent entraîner en retour des modifications obligeant ce dernier à faire une synthèse des deux propositions. Les échanges entre l'architecte et les services d'urbanisme relèvent d'un paradigme prescripteur / contrôleur : l'urbaniste devra prononcer un avis sur la conformité des plans de l'architecte aux règles d'urbanisme (Figure 1).

Figure 1 : Types de relations entre architecte et partenaires

(Source : Halin et al. $1998:$ 10).

Dans ces interactions, la coopération peut être forte mais elle n'induit pas nécessairement des rapports de négociation (les acousticiens ou les thermiciens ne négocient pas la solution architecturale ; les règles d'urbanisme ne sont pas, par nature, des objets négociables, etc.) À moins donc de distendre la notion de négociation à l'infini, les requêtes : « pour information », « pour addition » ou « pour validation » échappent au domaine de la négociation. 
Conclusion : l'engouement actuel à percevoir de la négociation et du compromis dans les interactions sociales appauvrit la description des rapports entre acteurs de la conception. À faire de la négociation un trait saillant, on oblitère d'autres types d'interactions sociales, qui semblent pourtant d'un niveau d'importance comparable dans les pratiques de conception.

\section{Les compétences spécifiques du concepteur}

Un autre trait des travaux récents de sociologie de la conception est de mettre en doute les notions de compétences et d'expertise professionnelle admises par la plupart des chercheurs sur la conception. Certains sociologues ont avancé que : « Cet intérêt pour la conception n'est pas neutre, il correspond peu ou prou au désir de légitimer l'action d'une profession » (Tapie, 2000, p. 15). D'autres interprètent l'approche cognitiviste de la conception comme un signe d'intérêt pour « la figure emblématique » de l'architecte, lequel ne mériterait pas un tel « traitement de faveur ». Il faudrait délaisser « le point de vue d'une création localisée [...] toute entière logée dans des compétences cognitives »(Callon, 1996, pp. 34, 27). Là encore, il semble que le questionnement de la notion de compétence prenne une forme maximaliste. On doit admettre que les compétences sont toujours révisables. La concurrence exercée par de nouvelles professions - les BET (bureaux d'études techniques), créés dans les années 1960 — ou par de nouveaux segments professionnels — les architectes spécialisés dans le DAO (dessin assisté par ordinateur), à partir des années 1980 — redistribue évidemment les compétences de la conception architecturale. Les évolutions récentes de la profession d'architecte avaient d'ailleurs été déjà pressenties dès les années 1970, par ceux qui envisageaient « l'éclatemment et la fragmentation de la mission traditionnelle » (Moulin et Lautman, 1978, p. 50), thème qui se trouve aujourd'hui largement repris par les travaux sur les ingénieries spécifiques (Prost, 1999) et sur l'évolution des compétences professionnelles (Champy, 1999). Beaucoup s'accordent à reconnaître que les NTIC (nouvelles technologies de l'information et de la communication) et les EDI (échanges de données informatisées) obligent à une révision de la dimension collaborative des tâches de conception architecturale. Les exigences croissantes de la maîtrise d'ouvrage et la forte compétitivité des professionnels coconditionnent une évolution des pratiques de conception en direction de la « concourance » (Midler et Jouini, 1996). Mais la question demeure de savoir comment passer d'un ajustement à la négation des compétences professionnelles, comme le font certains en affirmant la 
primauté absolue du réseau sur les concepteurs : «L'architecte n'existe pas » (Callon, 1996, p. 29).

\subsection{La notion de compétence et sa critique}

Il serait faux de croire que cette controverse sur les compétences résulte d'un partage entre internalistes - (Boudon, 1992, 1994, 1995 ; Conan, 1981, 1990 ; Prost, 1992, 1995) - et externalistes - (Toussaint et Younès, 1997 ; Champy, 1998, 1999 ; Tapie, 1999, 2000). En effet, certains travaux de sociologie de la conception, qui adoptent délibérément un point de vue externaliste, ne souscrivent pas à la thèse d'une absence des compétences. Guy Tapie reconnaît par exemple que l'architecte possède des « compétences génériques, à savoir ses capacités de création et de conception »(Tapie, 2000, p. 206). Il s'agit plutôt d'une controverse entre deux paradigmes sociologiques, qui réapparaît au sujet de la conception architecturale. Les notions mêmes de «compétence » et d'« expertise » sont issues de la sociologie des professions. Un article de synthèse rappelle que le concept de profession combine : « une compétence techniquement et scientifiquement fondée », et «l'acceptation et la mise en pratique d'un code éthique réglant l'exercice de l'activité professionnelle » (Chapoulie, 1973, p. 92). Il est clair que les auteurs de l'enquête sur Les architectes ont adhéré à des idées similaires, en avançant que l'identité de l'architecte est « fondée sur l'exclusivité d'une compétence résultant d'une formation spécifique » (Moulin et al., 1973, p. 30). Dans un article ultérieur, les auteurs qualifient même cette «expertise spécifique : la conception » (Moulin et Lautman, 1978, p. 51).

En marge de cette approche, le courant interactionniste est à l'origine d'une critique du concept de compétence. Mais cette critique est elle-même fort variable quant à son amplitude. On peut ainsi reconnaître que « Les frontières des compétences sont perpétuellement en débat » (Abbott, 1988, p. 2), parce qu'il existe une concurrence des groupes professionnels ou des segments d'une même profession à se les approprier. Il semble plus difficile d'abandonner purement et simplement le concept de compétence. Lorsque, dans la première partie d'une étude consacrée aux médecins, le concept de compétence est délaissé pour faire de la « profession » une notion caractérisant un « appétit de prestige » du professionnel (Freidson, 1984), l'auteur est condamné à analyser des faits d'un intérêt discutable en comparant le médecin au sorcier zandé d'Edward E. Evans-Pritchard (1937). L'un et l'autre tireraient leur 
pouvoir de leur tenue vestimentaire et de leur jargon inhabituels. Le problème de cette approche polémique de la compétence est que, partant d'un désir — louable — de ne pas établir de hiérarchie a priori entre les professions, elle est contrainte de faire une hypothèse beaucoup plus lourde : car l'asymétrie entre clients incompétents et professionnels compétents est alors troquée pour une asymétrie entre clients crédules et professionnels manipulateurs. On est en droit de se demander si la confiance que le patient voue au médecin émane de l'impression que lui causent blouse blanche et stéthoscope, ou si elle ne repose pas plutôt sur le savoir ordinaire que les études de médecine sont sanctionnées par un diplôme, et que l'exercice illégal de la médecine est condamné par la Loi.

\subsection{Le modèle de l'acteur-réseau appliqué à l'architecture}

La négation des compétences professionnelles pose des questions spécifiques dans le domaine de l'architecture. L'idée selon laquelle « l'architecte n'existe pas » semble se déduire d'une application du programme de l'acteur-réseau développé, en sociologie des sciences et techniques, par le Centre de Sociologie de l'Innovation de l'École des Mines de Paris ${ }^{10}$ (Latour, 1984, 1989 ; Callon, 1989). Ainsi, pour prendre un autre exemple échappant au domaine de l'architecture, le biochimiste Rose n'est pas un savant ; il n'est que le « porteparole » d'un réseau composé d'éléments hétérogènes : rats, seringues, éther, chronomètres, compteurs Geiger, tubes d'héparine, épreuves à corriger, demandes de crédits, etc. (Law, 1989). Sans ce réseau hybride (humain, non humain), Rose ne serait plus rien... Les idées récentes sur la profession d'architecte doivent beaucoup à cette conception de l'acteur-réseau. Certains sociologues (Callon, 1996, p. 28) ont demandé de « redistribuer le processus de conception sur une pluralité d'acteurs ", que ces acteurs soient humains (maître d'ouvrage, ingénieurs, économistes, sous-traitants, etc.) ou non humains (ordinateurs, plans, descriptifs, etc.) Chaque acteur étant nécessaire à la progression des études de conception, il n'existerait plus de raison d'accorder à l'architecte un «traitement de faveur ». Cette application du modèle de l'acteur-réseau à l'architecture soulève plusieurs difficultés.

Primo, cette position ne tient pas longtemps devant les besoins de l'analyse. Une fois

\footnotetext{
${ }^{10}$ Pour une critique des idées de l'acteur-réseau, à savoir : non-différenciation des activités humaines ; extension des connaissances du local au global, (Shinn, 2000, p. 464). D'autres problèmes de la nouvelle sociologie des sciences et techniques ont été soulevés, notamment (Boudon, 1990).
} 
affirmé que «L'architecte n'existe pas », la figure de l'architecte est réintroduite, et parfois même dans une perspective fonctionnaliste : «C'est le travail de l'architecte de réaliser cette inscription $^{11} »($ Callon, 1996, pp. 28, 30). Mais si l'« inscription » est bien un travail régulier de l'architecte, auquels ses partenaires s'attendent de lui, ne faut-il pas alors réintroduire aussi les compétences spécifiques qui sont mobilisées pour réaliser cette inscription ? La critique du cognitivisme à laquelle se livre ensuite l'auteur (Callon, 1996, pp. 34-35) prête le flanc à la même objection. Pour prétendre que l'analyse architecturologique « a retiré tous ses réseaux » à l'architecte Stanislas Fiszer, l'auteur est tenu de réunir acteurs réels et acteurs virtuels de la conception, en rattachant les seconds par le concept d'« envoi », emprunté à la sémiotique. Or il existe une différence fondamentale ${ }^{12}$ entre l'acteur réel (le maître d'ouvrage, par exemple) et l'acteur virtuel (le lycéen, que le concepteur imagine circuler dans le bâtiment) : c'est qu'il n'existe de négociation possible que dans le premier cas. Dans le second cas, il s'agit de tout autre chose : l'« envoi » de l'architecte dans l'usager du bâtiment constitue une tâche de conception, que ni le maître d'ouvrage, ni l'ingénieur, ni le contrôleur technique, ni a fortiori l'ordinateur, ne peuvent prendre en charge. L'envoi, qui signale un travail de représentation de la praticabilité des espaces, témoigne d'une «capacité cognitive » spécifique de l'architecte (Deshayes, 1999). Il est donc surprenant que ce concept puisse servir de fondement à une critique des compétences du concepteur.

Secundo, l'application du programme de l'acteur-réseau à l'architecture tend à récuser toute spécificité aux acteurs de la conception : «Ce que fait l'architecte [...] n'est pas différent de ce que fait le collectif auquel participe le technicien, le porte-parole du lycéen, l'ordinateur qui va chercher dans un catalogue mis en mémoire les modèles de poutres disponibles ou les formes déjà imaginées pour d'autres lycées »(Callon, 1996, pp. 34-35). Cette mise à plat de tous les acteurs n'est pas sans poser problème.

1) Entre humains et non-humains, tout d'abord. Car le fait bien connu, que l'informatique

\footnotetext{
${ }^{11}$ Il s'agit de l'inscription dans le bâtiment de points de vue et de contraintes diverses, comme le veut la notion d'« ingénierie hétérogène » (Law, 1987).

${ }^{12}$ Cette différence est pointée par Philippe Deshayes, lorsqu'il fait une distinction entre l'« usager routinier » et l'« usager virtuel », par exemple : la famille à laquelle est destinée une maison d'habitation versus les visiteurs potentiels de cette maison (Deshayes, 1999, p. 14). On retrouve le même trait : seuls les premiers destinataires pourront prendre part à une négociation, les seconds n'étant susceptibles que d'une représentation mentale, au moment de la conception.
} 
appliquée à l'architecture reste cantonnée aux tâches de DAO, montre que l'ordinateur n'est pas en mesure de faire ce que fait le plus mauvais des architectes, à savoir : concevoir un bâtiment qui réponde satisficing à un ensemble de contraintes économiques, fonctionnelles, techniques, etc.

2) Entre humains, ensuite. Car si l'on prend à la lettre l'idée qu'il n'est pas de traitement de faveur ni de compétences spécifiques, on devrait alors pouvoir substituer les positions respectives de tous les acteurs du réseau : l'économiste du bâtiment devenant ingénieur structure, le maître d'ouvrage devenant architecte, etc. L'expérience - menée par un maître d'ouvrage au grand cœur - démontrerait qu'un tel échange de positions est inopérant, parce que l'exercice de la profession d'ingénieur ou d'architecte est le fruit d'un apprentissage durant lequel un individu a acquis des compétences professionnelles spécifiques. Ces compétences ne peuvent pas être niées. Au-delà du titre professionnel, est ingénieur structure qui connaît la différence entre un système isostatique et un système hyperstatique, les bulbes de compression de Kögler-Scheidling, le moment d'inertie d'une poutre IPN 180-82, etc. Est architecte qui connaît la formule de Blondel, le principe de calcul de l'azimut et de la hauteur du soleil à une date et à une latitude données, le niveau d'éclairement nécessaire dans un bureau, le système d'établissement des dimensions nominales des baies, l'article R. 111-17 du Code de l'Urbanisme, etc. Ces connaissances sont évidemment accessibles à tous, mais les rapports de confiance qui s'établissent entre le maître d'ouvrage, l'architecte ou l'ingénieur structure naissent, plus que de la persuasion, de l'attente légitime que tel expert doit normalement posséder tel ensemble de connaissances spécifiques. Certains auteurs, travaillant dans une cadre proche du modèle négocié, tirent d'ailleurs une conclusion différente au sujet des compétences professionnelles de l'architecte: «L'efficacité de la co-conception ne peut se substituer à l'efficacité propre de chacun des intervenants. Au contraire, elle ne fait que mieux mettre en avant la compétence propre de chacun des acteurs et leurs responsabilités dans le projet » (Bignon et al., 1999).

Conclusion : le modèle de l'acteur-réseau tend à homogénéiser le réseau, en concevant les relations (arcs du réseau) sous l'exclusive de la négociation, et en ramenant les acteurs (sommets du réseau) à des noeuds formels auxquels on a retiré toute compétence professionnelle. 


\section{Conception architecturale / conception industrielle}

Les trois sous-problèmes étudiés dans les sections précédentes soulignent l'échec d'une transposition des résultats de l'anthropologie des sciences et des techniques à l'architecture, en dépit de la rhétorique qui tend à la présenter comme « une analyse empirique » «plus réaliste »(Callon, 1996, pp. 26, 32). Il convient de s'interroger sur les raisons de cet échec. Elles semblent être en rapport avec la sous-évaluation de la spécificité des tâches de conception en architecture. La conception d'un objet technique — qui sert de modèle d'approche de l'architecture - et la conception d'un bâtiment diffèrent sur un grand nombre de points, qui peuvent servir de canevas d'une analyse comparative.

\subsection{Trois différences principales}

Nous commencerons par mettre en évidence quelques traits distinctifs des situations de conception architecturale versus de conception industrielle, avant de voir comment ces traits distinctifs induisent des modèles d'organisation de conception différents.

4.1.1. Les objets industriels sont produits en série, les bâtiments sont des exemplaires uniques. C'est le cas si l'on considère une liste d'objets courants : un ventilateur AEG, un Minitel 3 de France Télécom, un verrou de sécurité Cavers, un dérailleur électrique Mavic, un catamaran New Marine sont des produits de série, alors que, de la petite villa Coucou du Team Zoo à la pyramide du Grand Louvre, les bâtiments sont des objets uniques. Certains objets ont un statut plus ambigu, comme le métro Aramis de Matra, resté à l'état de prototype, ou le coronographe-spectographe de Jacques-Clair Noëns de l'observatoire du Pic du Midi, qui n'a jamais eu vocation à être reproduit. Toutefois, c'est ici le niveau d'usage de ces dispositifs techniques qui fixent leur unicité. Si les détecteurs de particules, les coronographes ou les polarimètres K étaient des objets d'un usage plus répandu, ils se prêteraient sans difficulté à une production en série. Au contraire, les bâtiments restent des objets uniques, quoiqu'ils soient des objets usuels de la vie quotidienne.

\subsubsection{Les objets industriels sont identifiés par leur marque, les édifices sont identifiés par leur localisation.} Gela a une conséquence immédiate sur la procédure de recherche des responsabilités. Imaginons qu'un appareil technique tombe en panne et qu'un bâtiment prenne l'eau, à cause d'un vice de conception. Dans le premier cas, c'est par la marque apposée sur l'objet que l'on trouvera le responsable de la défaillance. Dans le second, c'est par le syndic de l'immeuble. En 
outre, le simple fait que la marque soit apposée sur l'objet technique révèle une différence de statut des concepteurs. Dans le premier cas, le concepteur est employé par la firme qui a fabriqué l'objet. C'est donc la firme (et non le concepteur) qui, s'étant livrée à une activité commerciale, endossera la responsabilité de la défaillance. Dans le second cas, l'architecte ne vend pas l'édifice et n'est pas salarié par le maître d'ouvrage. Exerçant une profession libérale, il n'a qu'une mission de conseil auprès de ce dernier. Les responsabilités devront alors être étudiées au cas par cas, et seront en général redistribuées selon les domaines respectifs d'expertise des concepteurs, des constructeurs et des fournisseurs.

4.1.3. Les objets industriels sont des meubles, les édifices sont des immeubles (ce sont des productions situées). Ce trait est patent, même dans le cas des objets techniques au statut ambigu, comme le métro Aramis de Matra, que l'on a successivement envisagé d'implanter à Paris, Montpellier ou Orly. D'une façon comparable, la chambre de Wilson du cosmicien Patrick Blackett a été déplacée du laboratoire de Manchester jusqu'à l'Observatoire du Pic du Midi en 1949, en dépit du poids propre de son électro-aimant (environ 4 tonnes). Cette dernière propriété distinctive - meuble versus immeuble - est plus fondamentale que les précédentes, car elle autorise leur reconstruction.

Primo, la distinction meuble versus immeuble explique pourquoi le bâtiment ne porte pas de marque : son immobilité est la meilleure garantie que l'on puisse retrouver le responsable d'éventuels vices de conception.

Secundo, quoique la reconstruction de cette propriété soit plus longue, la distinction meuble versus immeuble justifie aussi pourquoi un bâtiment est une production unique : celui-ci doit répondre à une implantation dans un site donné. L'étude détaillée des contraintes de la conception architecturale montre que l'architecte doit prendre en considération deux types de contrainte : les contraintes dépendant du type d'édifice - IGH (immeuble de grande hauteur), ERP (édifice recevant du public), lieu de travail, etc. - et les contraintes de site - POS (plan d'occupation des sols), plan d'alignement, gabarits urbains, etc. Or, trois contraintes sur quatre sont des contraintes de site (Raynaud, à paraître). Répondant à de nombreuses contraintes de site, et les sites étant toujours différents les uns des autres, les bâtiments ne sont pas reproductibles à l'identique. Les bâtiments sont donc des produits uniques. Considérons par exemple l'hôtel «Le Belvédère » de Cerbère, implanté sur une parcelle triangulaire, pentue, et laissant passage sur l'arrière à la voie de chemin de fer 
Perpignan / Port Bou. Même si l'hôtel pouvait être reproduit ou déplacé à coût nul, cette reproduction ou ce déplacement ne présenterait aucun intérêt : car il est improbable que l'on trouve une deuxième parcelle ayant exactement les mêmes caractéristiques et les mêmes dimensions que la première.

La même remarque peut être faite à propos du Zollamtsbrücke de Vienne, pont conçu par Otto Wagner et ses assistants vers 1898 (Zednicek, 1995). Cet ouvrage a la particularité d'être un pont de métro en partie basse, et une passerelle en partie haute. Le pont et la passerelle ne sont pas parallèles entre eux, et chacun forme un angle différent par rapport à l'axe du canal. La conception de l'ouvrage a dû prendre en considération : a) le niveau des eaux, b) le niveau des rails, c) le niveau des berges, d) la hauteur des wagons du métro, e) l'écartement des berges, f) l'angle $\alpha$ de la voie du métro par rapport au canal, g) l'angle $\beta$ de la passerelle par rapport au canal, etc. C'est pourquoi il n'est pas envisageable que l'ouvrage, en raison des contraintes de site spécifiques auxquelles il répond, puisse être implanté ailleurs que là où il est installé.

Ce trait distinctif - meuble versus immeuble - permet donc bien une reconstruction des deux premiers (objets produits en série versus exemplaires uniques ; objets identifiés par la marque versus identifiés par leur localisation).

\subsection{Retour sur les modèles d'organisation}

Essayons maintenant de rapprocher ce trait - meuble versus immeuble — des modèles d'organisation qui prévalent dans l'industrie et en architecture. Sachant que les bâtiments sont soumis à un grand nombre de contraintes de site, sachant que les contraintes sont le point de départ de l'activité de conception, celle-ci consistant à imaginer des solutions satisficing, la spécificité de la mission de conception de l'architecte - et par voie de conséquence, une dimension irréductible de son expertise - consiste à se saisir de l'ensemble des contraintes (dont les contraintes de site) pour soumettre une proposition architecturale au maître d'ouvrage.

C'est donc parce que les contraintes de site sont connaissables ex ante qu'il peut exister en architecture un travail de conception préalable à toute consultation des autres concepteurs. En effet, ni l'ingénieur structure, ni l'économiste du bâtiment, ni le thermicien, ni l'acousticien, etc. ne sont habilités à donner une réponse architecturale à cet ensemble de contraintes 
spécifiques. L'architecte bénéficie d'une avance sur les autres concepteurs dans la connaissance du projet. Cette propriété est sans équivalent dans la conception des objets industriels, pour laquelle les contraintes de site sont inexistantes ou extrêmement limitées. Les concepteurs se trouvent alors plus facilement sur un pied d'égalité dans l'approche du projet, et peuvent formuler des propositions qui se ressentent moins d'une compartimentalisation des tâches. Aucun n'a d'avance sur l'autre. C'est la raison pour laquelle le «modèle négocié », qui prévaut dans la conception industrielle, reste - malgré des encouragements constants — difficilement transposable en architecture, tout au moins sur la totalité de la phase de conception et entre tous les acteurs de la conception. Rien n'empêche que cette situation puisse évoluer, le partage des expertises étant évidemment révisable, mais il restera toujours le problème insoluble que les contraintes de site induisent un niveau d'information différent entre le concepteur principal (architecte) et les concepteurs secondaires (ingénieur structure, thermicien, acousticien, etc.)

\section{Conclusion}

Si l'on doit admettre l'existence du phénomène de dérive, une part de négociation dans la conception et le caractère révisable des compétences professionnelles, la maximalisation de ces conclusions conduit à des résultats peu conformes au domaine de la conception architecturale. La dérive n'est pas un phénomène inéluctable ; l'accent mis sur la négociation occulte d'autres rapports sociaux fondamentaux; les compétences des acteurs ne sont pas interchangeables à merci. L'échec de la transposition résulte d'un trait essentiel de la conception architecturale : concevoir des objets immeubles situés. C'est pourquoi, en définitive, et contrairement à ce qu'affirment certains, ce que fait l'architecte est non seulement différent de ce que fait le géomètre, le porte-parole du lycéen, ou l'ordinateur, qui n'ont pas à concevoir, mais encore différent de ce que fait l'ingénieur structure, l'acousticien ou le thermicien, qui n'ont pas pour mission d'élaborer une proposition répondant à des contraintes connues ex ante.

Il faut donc revenir à cette proposition un peu banale, jadis énoncée par les auteurs de l'enquête sur les architectes (Moulin et al., 1973), que la conception constitue le noyau dur de l'expertise professionnelle de l'architecte. Et si l'on devait définir le contenu de cette expertise, on devrait y mettre : a) la capacité d'analyse des contraintes de site du projet ; b) la capacité à formuler une proposition architecturale répondant satisficing aux contraintes du projet ; c) la capacité à imaginer, par le phénomène de l'« envoi », la réaction des usagers au bâtiment 
virtuel. En tous les cas, une sociologie de la conception architecturale ne saurait transposer des résultats élaborés sur d'autres objets, sans une analyse préalable des données et contraintes spécifiques de la conception en architecture.

\section{Références}

Abbott, A., 1988. The system of the professions ; an essay on the division of expert labour. Chicago University Press, Chicago.

Alexander, C., 1971. De la synthèse de la forme. Dunod, Paris [1964].

Bignon, J.-C., Malcurat, O., Halin, G., 1999. Coopération et conception. 6e Table ronde sur la conception de nouveaux systèmes d'information. Saint-Ferréol, 14-15 décembre 1999.

Boudon, Ph., 1992. Introduction à l'architecturologie. Dunod, Paris.

Boudon, Ph., Deshayes, Ph., Pousin, F., Schatz, F., 1994. Enseigner la conception architecturale. Éditions de la Villette, Paris.

Boudon, Ph., 1995. Existe-t-il des opérations de conception architecturale ? Prost, R. (éd.) Concevoir, inventer, créer. L'Harmattan, Paris, 259-276.

Boudon, R., 1990. L'art de se persuader des idées douteuses, fragiles ou fausses. Fayard, Paris.

Burns, T., Stalker, G., 1966. The management of innovation. Tavistock, London.

Callon, M. (éd.), 1989. La science et ses réseaux, genèse et circulation des faits scientifiques. La Découverte, Paris.

Callon, M., 1996. Le travail de la conception en architecture. Cahiers de la Recherche architecturale, 37, 25-35.

Callon, M., 1997. Concevoir : modèle hiérarchique et modèle négocié. Stoa, 2, 88-94.

Callon, M., Latour, B. (éds.), 1991. La science telle qu'elle se fait. La Découverte, Paris.

Canguilhem, G., 1968. Études d'histoire et de philosophie des sciences. Librairie philosophique J. Vrin, Paris.

Champy, F., 1988. Les architectes et la commande publique. PUF, Paris.

Champy, F., 1999. L'évolution des compétences des architectes en France depuis les années 80. Cahiers de la Recherche architecturale et urbaine, 2/3, 27-38.

Chapoulie, J.-M., 1973. Sur l'analyse sociologique des groupes professionnels. Revue française de Sociologie, 14, 86-114.

Conan, M., 1981. Les processus de conception architecturale. Plan Construction, Paris.

Conan, M., 1990. Concevoir un projet d'architecture. L'Harmattan, Paris. 
Conan, M., 1995. Les jeux imbriqués de la conception architecturale. Prost, R. (éd.) Concevoir, inventer, créer. L'Harmattan, Paris, 153-188.

Cuff, D., 1982. The context for design : six characteristics. Environmental Design Research Association, 13, 3847.

Deshayes, Ph., 1999. Cognition, communication et échanges dans les processus de conception. Compétences et langages de la conception chez les professionnels du projet. Rapport d'activité LAREA, Paris, 6-87.

Evans-Pritchard, E.E., 1937. Witchcraft, oracles and magic among the Azande. Oxford University Press, Oxford.

Freidson, E., 1984. La profession médicale. Payot, Paris [1970].

Guillot, X., 1989. Shin Takamatsu ; projets d'architecture. Electa Moniteur, Paris.

Halin, G., Benali, K., Bignon, J.-C., Godart, C., 1998. Cooperation models in co-design. 4th International conference on design and decision support systems in architecture and urban planning. Maastricht, July 2629, 1998.

Latour, B., 1984. Les microbes, guerre et paix ; irréductions. Métailié et Pandore, Paris.

Latour, B., 1989. La science en action. La Découverte, Paris.

Law, J., 1987. Technology and heterogeneous engineering. Bijker, W. (ed.) The social construction of technology. The MIT Press, Cambridge.

Law, J., 1989. Le laboratoire et ses réseaux. Callon, M. (éd.). La science et ses réseaux, genèse et circulation des faits scientifiques. La Découverte, Paris, 117-148.

Lebahar, J.-C., 1983. Le dessin d'architecte ; simulation graphique et réduction d'incertitude. Parenthèses, Marseille.

Malcurat, O., Bignon, J.-C., Halin, G., 2000 a. Improving cooperation in small scale projects. 8th International conference in civil and building engineering. Stanford University, August 14-17, 2000.

Malcurat, O., Bignon, J.-C., Halin, G., Hanser, D., 2000 b. CoCAO : Collecticiel à l'usage des métiers du bâtiment. Conférence IBPSA'2000. Sophia-Antipolis, 26-27 octobre 2000.

Midler, Ch., Jouini, S.B.M., 1996. L'ingénierie concourante dans le bâtiment ; synthèse des travaux du GREMAP. Éditions du Plan Construction Architecture, Paris.

Moulin, R., Gros, P., Lautman, J., Martinon, J.-P., Schnapper, D., 1973. Les architectes ; métamorphoses d'une profession libérale. Calmann-Lévy, Paris.

Moulin, R., Lautman, J., 1978. Les architectes ; la profession libérale et son aggiornamento. Cahiers de la Recherche architecturale, 2, 49-55. 
Neuberg, M., 1990. La contrainte. Dialogue, 29, 491-522.

Nozick, R., 1991. La contrainte. Neuberg, M. (éd.) Théorie de l'action. Mardaga, Liège, 271-310 [1969].

Pfeiffer, B.B., 1990. Frank Lloyd Wright drawings. Harry N. Abrams Inc, New York.

Prost, R., 1992. Conception architecturale : une investigation méthodologique. L'Harmattan, Paris.

Prost, R. (éd.), 1995. Concevoir, inventer, créer ; réflexions sur les pratiques. L'Harmattan, Paris.

Prost, R., 1999. Les pratiques architecturales en mutation. Cahiers de la Recherche architecturale et urbaine, 2/3, 85-94.

Raynaud, D., 1999. Le schème, opérateur de la conception architecturale. Intellectica, 29, 35-69.

Raynaud, D., à paraître. Les contraintes de la conception architecturale.

Reynaud,J.-D., 1999. Le conflit, la négociation et la règle. Octarès, Toulouse.

Riley, T., Reed, P. (eds.), 1994. Frank Lloyd Wright architect. MoMA, New York.

Shinn, T., 1980. Division du savoir et spécificité organisationnelle ; les laboratoires de recherche industrielle en France. Revue française de Sociologie, 21, 3-35.

Shinn, T., 2000. Formes de division du travail scientifique et convergence intellectuelle ; la recherche technicoinstrumentale. Revue française de Sociologie, 41, 447-473.

Simon, H.A., 1974. Sciences des systèmes, sciences de l'artificiel. Epi, Paris [1963].

Simounet, R., 1996. Roland Simounet ; pour une invention de l'espace. Electa Moniteur, Milan / Paris.

Strauss, A., 1992. La trame de la négociation ; sociologie qualitative et interactionnisme. Textes réunis par I. Bazsanger. L'Harmattan, Paris.

Tapie, G., 1999. Professions et pratiques. Cahiers de la Recherche architecturale et urbaine, 2/3, 65-74.

Tapie, G., 2000. Les architectes : mutations d'une profession. L'Harmattan, Paris.

Tata, S., Canals, G., Godart, G., 1999. Specifying interactions in cooperative applications. 11 th International conference on software engineering and knowledge engineering. Kaiserlautern, 17-19 juin 1999.

Thuderoz, C., 2000. Négociations ; essai de sociologie du lien social. PUF, Paris.

Toussaint, J.-Y., Younès, G. (éds.), 1997. Architecte, ingénieur ; des métiers et des professions. Éditions de la Villette, Paris.

Tric, O., 1999. Conception et projet en architecture. L'Harmattan, Paris.

Zednicek, W., 1995. Otto Wagner, Adolf Loos, Josef Hoffmann, Wagner-Schüler, Wien Architecktur. Bundesministeriums für Unterricht und Kunst, Wien. 